\title{
L'assurance-invalidité lance deux projets de recherche sur la collaboration avec les médecins traitants et la procédure d'instruction dans l'AI
}

\section{Stefan Ritler}

Vice-directeur, chef du domaine Assurance-invalidité,

Office fédéral des assurances sociales OFAS
Les appels d'offre pour ces deux projets sont consultables, jusqu'au délai de dépôt des offres (22 juillet 2013), à l'adresse www.bsv.admin.ch/ praxis/forschung/00105/ index.html?lang=fr.

Correspondance:

Stefan Ritler

Département fédéral de

l'intérieur DFI

Office fédéral des assurances sociales OFAS

Effingerstrasse 20

CH-3003 Berne

Tél. 0313229215

Fax 0313223715
Les médecins traitants sont des partenaires importants de l'assurance-invalidité (AI). Pour elle, il est essentiel d'intervenir rapidement lorsqu'une personne voit son emploi compromis par des problèmes de santé, afin de renforcer ses ressources et d'éviter autant que possible une longue période d'incapacité de travail. Personnes de référence et de confiance des assurés, dont ils connaissent parfaitement les antécédents médicaux et les possibilités de traitement, les médecins traitants peuvent contribuer de façon déterminante au succès de la réadaptation de leurs pa- s'exerce: les mesures médicales destinées aux personnes atteintes d'infirmités congénitales, les mesures d'instruction axées sur la réadaptation, l'examen approfondi du droit à la rente et la procédure de révision des rentes. En plus de présenter les formes de collaboration et d'identifier facteurs de réussite et obstacles, le projet doit aussi sensibiliser les deux acteurs aux facteurs propices à la collaboration, contribuant par là-même à améliorer celle-ci. En ce sens, il a une visée formatrice et doit favoriser les processus d'apprentissage. Un appel d'offres est en cours.

\section{«Les médecins traitants peuvent contribuer de façon déterminante} au succès de la réadaptation de leurs patients.»

tients; ils les accompagnent dans ce processus après avoir soutenu les offices AI par leur appréciation du potentiel de réadaptation présenté. La collaboration entre offices AI et médecins traitants fait l'objet de deux projets de recherche lancés par l'AI.

\section{Projet de recherche: «La collaboration entre offices Al et médecins traitants: formes, instruments et appréciation par les acteurs»} Enjeu de taille tant pour les médecins traitants que pour l'AI, cette collaboration comporte des difficultés, mais conduit aussi à des succès communs. Ce projet vise à présenter les formes et instruments actuels de la collaboration entre offices AI et médecins traitants, et à identifier les facteurs de réussite et les obstacles à la collaboration. Les médecins installés, les sociétés médicales et le personnel des offices AI seront également invités à donner leur avis sur les questions suivantes: «Quelle est, en m'engageant dans cette collaboration, ma conception des rôles respectifs? Quelles sont mes attentes vis-à-vis de mon partenaire? Comment la collaboration pourrait-elle être améliorée?» On distingue quatre domaines où cette collaboration

\section{Projet de recherche: «La procédure d'instruction de l'assurance-invalidité pour les décisions d'octroi de rente: processus, acteurs et effets»}

Le second projet mis au concours concerne lui aussi les médecins traitants. Il porte sur les mesures d'instruction médicale et professionnelle pilotées par les offices $\mathrm{AI}$ dans le processus de décision en matière de rentes: comment les offices AI organisent-ils l'instruction? Quelles manières typiques de procéder peut-on distinguer? Quel est l'impact des différentes manières de procéder, par exemple sur la durée et les coûts de la procédure d'instruction, ou sur la probabilité d'un recours devant le tribunal? L'objectif de l'étude est d'identifier et d'analyser les procédures des offices AI tant au niveau stratégique que pratique en matière d'instruction médicale et professionnelle, ainsi que leur efficacité. Les repères acquis grâce à cette étude doivent avant tout former une base en vue d'optimiser l'instruction AI et contribuer à une meilleure collaboration avec les divers acteurs, et notamment les centres d'expertises médicales, les centres d'observation professionnelle et les médecins traitants ${ }^{1}$. 